\section{Recomendaciones para el tratamiento de infecciones por cocos grampositivos en pacientes críticos. De la teoría a la práctica}

\section{Sr. Director:}

En primer lugar quisiéramos agradecer a M. Gerónimo-Pardo sus palabras de felicitación por la publicación de las «Recomendaciones GEIPC-SEIMC y GTEI-SEMICYUC para el tratamiento antibiótico de infecciones por cocos grampositivos en el paciente crítico» ${ }^{1,2}$. La elaboración de unas recomendaciones basadas en la evidencia y en la experiencia de los participantes no es tarea fácil ya que es necesario sintetizar y consensuar aquellas prácticas sobre las que existe mayor información contrastada. Los comentarios incluidos en su carta abordan aspectos farmacológicos, especialmente sobre vancomicina, que han sido considerados en nuestro artículo, aunque es posible que no con la intensidad necesaria para clarificar nuestra posición, por lo que complementamos cada uno de ellos a continuación:

1. Dosis de carga: en la actualidad existe controversia acerca del índice farmacocinético-farmacodinámico $(\mathrm{Pk} / \mathrm{Pd})$ que mejor puede predecir el resultado terapéutico del tratamiento con vancomicina. Mientras que algunos autores han considerado la vancomicina como un antibiótico tiempo-dependiente $^{3}$, en la actualidad se ha propuesto que la relación entre el área bajo la curva y la concentración mínima inhibitoria (AUC/CMI) podría comportarse como un mejor índice $\mathrm{Pk} / \mathrm{Pd}$ de eficacia terapéutica ${ }^{4}$. Independientemente del índice $\mathrm{Pk} / \mathrm{Pd}$ que se utilice en estudios de investigación, en la práctica clínica es recomendable alcanzar concentraciones de vancomicina en rango terapéutico lo antes posible. Las bajas concentraciones de vancomicina se han relacionado con la emergencia de cepas resistentes, mientras que la administración de una terapia precoz con dosis más elevadas se ha relacionado con resultados más satisfactorios $^{5}$. De esta forma, podría pensarse que la dosis de carga, utilizada como una estrategia para alcanzar rápidamente concentraciones terapéuticas (como en el caso de teicoplanina), debería aplicarse cuando se utiliza vancomicina. Mientras esta práctica (dosis de carga) se recomienda en nuestro artículo para los casos en los que se decide el empleo de vancomicina en perfusión continua (pág. 296, segunda columna, tercer párrafo, línea 5), y especialmente cuando es necesario alcanzar elevadas concentraciones plasmáticas (pág. 299, primera columna, tercer párrafo, línea 12), no se recomienda cuando su administración es intermitente. Incrementar la dosis el primer día, entre un $50-100 \%$ respecto a la dosis de mantenimiento, como sugiere Gerónimo-Pardo, no está evaluado en el entorno del paciente crítico y, por el contrario, existen datos que sugieren una mayor toxicidad cuanto mayor es la dosis administrada y la concentración plasmática alcanzada 5 . Deberían existir estudios experimentales y/o clínicos aleatorizados que apoyarán esta práctica para poder incluir la dosis de carga de vancomicina, cuando se administra de 
forma intermitente, en unas recomendaciones terapéuticas avaladas por sociedades científicas.

2. Individualización del tratamiento: es evidente que en un paciente crítico deben individualizarse las dosis de los fármacos administrados, incluidos los antimicrobianos, y así lo expresamos en las recomendaciones publicadas (pág. 298, primera columna, segundo párrafo, línea 1). Las dosis propuestas en la tabla 3 están individualizadas atendiendo al aclaramiento de creatinina y desconocemos si existe otra forma de hacerlo, en el entorno del paciente crítico, atendiendo a otros parámetros (situaciones hiperdinámicas, edad).

3. Infradosificación relativa: uno de los riesgos el utilizar antimicrobianos, en pacientes críticos, a dosis fijas (de acuerdo con la ficha técnica), es la infradosificación relativa (niveles plasmáticos infraterapéuticos), con las consecuencias clínicas ya comentadas. Esto es especialmente frecuente en pacientes con función renal normal y estado hiperdinámico, ya sea por su patología de base, ya sea por los fármacos administrados. Esta situación es especialmente relevante en los casos de infección del sistema nervioso central, en los que se recomienda alcanzar niveles plasmáticos de antibióticos más elevados. Por ese motivo se ha incluido en las recomendaciones la obligatoriedad de monitorizar las concentraciones plasmáticas en el valle, para mantener niveles de vancomicina entre 20$30 \mathrm{mg} / \mathrm{ml}$ (pág. 299, primera columna, párrafo 3, línea 3). Lamentablemente la monitorización de concentraciones plasmáticas de antibióticos no está suficientemente estandarizada en la práctica diaria de los hospitales españoles, existiendo importantes diferencias en su aplicabilidad ${ }^{6}$.

4. Nefrotoxicidad de vancomicina: en las recomendaciones publicadas por nosotros se especifica que posiblemente la toxicidad de vancomicina está sobreestimada (pág. 296, segunda columna, segundo párrafo, línea 4), sin embargo esta no es nula, como parece indicar Gerónimo-Pardo en sus comentarios. No existe ningún estudio que conozcamos que cuando analiza la morbilidad relacionada con vancomicina no aporte diferentes tasas de toxicidad renal. Asimismo existen estudios que demuestran mayor toxicidad renal cuanto mayor es la concentración plasmática alcanzada ${ }^{5,7}$. Por ello, su afirmación de no limitar los rangos máximos de los niveles plasmáticos «por miedo a una toxicidad que no es tal» debe tomarse con cautela, y en la práctica clínica deben seguirse las recomendaciones sobre las que existen evidencias demostradas. En todo caso, sería de gran ayuda, para evaluar su afirmación en su medida, disponer de los datos de uso de vancomicina en su unidad, así como de los criterios seguidos para incrementar las dosis de vancomicina.

P.M. OLAECHEA-ASTIGARRAGA ${ }^{\mathrm{a}}$, S. GRAU CERRATO ${ }^{\mathrm{b}}$, F. ÁLVAREZ-LERMA ${ }^{c}$ Y J. GARNACHO-MONTERO ${ }^{d}$ (EN NOMBRE DE LOS AUTORES) ${ }^{a}$ Servicio de Medicina Intensiva. Hospital de Galdakao. Vizcaya. ${ }^{b}$ Servicio de Farmacia. Hospital Universitario del Mar. Barcelona. ${ }^{c}$ Servicio de Medicina Intensiva. Hospital Universitario del Mar. Barcelona. ${ }^{d}$ Servicio de Medicina Intensiva y Urgencias. Hospital Virgen del Rocío. Sevilla. España.

\section{BIBLIOGRAFÍA}

1. Olaechea Astigarraga P, Garnacho Montero J, Grau Cerrato S, Rodríguez Colomo O, Palomar Martínez M, Zaragoza Crespo $\mathrm{R}$, et al. Recomendaciones GEIPC-SEIMC y GTEI-SEMICYUC para el tratamiento antibiótico de infecciones por cocos grampositivos en el paciente crítico. Med Intensiva. 2007;31:294-317.

2. Olaechea Astigarraga P, Garnacho Montero J, Grau Cerrato S, Rodríguez Colomo O, Palomar Martínez M, Zaragoza Crespo $\mathrm{R}$, et al. Recomendaciones GEIPC-SEIMC y GTEI-SEMICYUC para el tratamiento antibiótico de infecciones por cocos grampositivos en el paciente crítico. Enferm Infecc Microbiol Clin. 2007; 25:446-66.

3. Ross GH, Wright DH. Glycopeptide pharmacodynamics. En: Nightingale CH, Murakawa T, Ambrose PG, editors. Antimicrobial pharmacodynamics in theory and clinical practice. New York: Marcel Dekker; 2002. p. 177-204.

4. Mohr JF, Murray BE. Point: Vancomycin is not obsolete for the treatment of infection caused by methicillin-resistant Staphylococcus aureus. Clin Infect Dis. 2007;44:1536-42.

5. Hidayat LK, Hsu DI, Quist R, Shriner KA, Wong-Beringer A. High-dose vancomycin therapy for methicillin-resistant Staphylococcus aureus infections: efficacy and toxicity. Arch Intern Med. 2006; 166:2138-44.

6. Álvarez Lerma F, Grau S, Marín-Sacino M, Olaechea P, Sánchez M, Martín E, et al, y GEIPC-SEIMC. Monitorización de concentraciones plasmáticas de antibióticos en hospitales españoles. Enferm Infecc Microbiol Clin. 2006;24:14-9.

7. Jeffres MN, Isakow W, Doherty JA, Mick ST, Kollef MH. A retrospective analysis of possible renal toxicity associated with vancomycin in patients with health care-associated methicillin-resistant Staphylococcus aureus pneumonia. Clin Ther. 2007;29: 1107-15. 